\title{
The C-terminus of MIP-T3 protein is required for ubiquitin-proteasome-mediated degradation in human cells
}

Chao-Wan Guo ${ }^{\mathrm{a}}$, Ge Liu ${ }^{\mathrm{a}}$, Sheng Xiong ${ }^{\mathrm{a}, \mathrm{b}}$, Feng Ge ${ }^{\mathrm{a}}$, Takayuki Fuse ${ }^{\mathrm{a}, \mathrm{c}}$, Yi-Fei Wang ${ }^{\mathrm{b}}$, and Kaio Kitazato ${ }^{\text {a* }}$

a. Division of Molecular Pharmacology of Infectious Agents, Department of Molecular Microbiology and Immunology, Graduate School of Biomedical Sciences, Nagasaki University, Nagasaki, Japan.

b. Biomedical R\&D Center, Guangdong Provincial Key Laboratory of Bioengineering Medicine, National Engineering Research Center of Genetic Medicine, Jinan University, Guangzhou, PR, China.

c. Division of Cellular and Molecular Biology, Department of Molecular Microbiology and Immunology, Graduate School of Biomedical Sciences, Nagasaki University, Nagasaki, Japan.

*Corresponding author. Tel: +81 95819 2457; Fax: +81 958192898

E-mail address: kkholi@nagasaki-u.ac.jp (Kaio Kitazato).

Postal address: Division of Molecular Pharmacology of Infectious Agents, Department of Molecular Microbiology and Immunology, Graduate School of Biomedical Sciences, Nagasaki University, 1-14 Bunkyo-Machi, Nagasaki 852-8521, Japan. 


\begin{abstract}
Intraflagellar transport (IFT) complex is essential for the formation and functional maintenance of eukaryotic cilia which play a vital role in development and tissue homeostasis. However, the biochemical characteristics and precise functions of IFT proteins remain unknown. Here, we report MIP-T3, a human microtubule-interacting protein that has been recently identified as a novel conserved component of IFT complex, is an easily degradable protein in human cell lines. The protein degradation is mediated by the ubiquitin-proteasome system, and its C-terminal is required for ubiquitination and proteasome-mediated degradation of MIP-T3 protein. This study provides the first evidence for regulation of IFT protein stability.
\end{abstract}

\title{
Key Words:
}

MIP-T3, intraflagellar transport, polyubiquitination, proteasome, protein ability 


\section{Introduction}

MIP-T3 (microtubule-interacting protein associated with TRAF3), also termed as TNF Receptor-Associated Factor 3 Interacting Protein 1 (TRAF3IP1), has been first identified as a human protein that binds to tubulin/microtubules and TRAF3 [1], IL-13 receptor alpha1 [2], and Disrupted-in-Schizophrenia 1 (DISC 1) [3]. Recently, several genome- and proteome-wide studies in different organisms have demonstrated that MIP-T3/TRAF3IP1 is a novel conserved component of intraflagellar transport (IFT) complex, which is essential for the formation and functional maintenance of eukaryotic cilia [4-8], and more intriguingly, IFT system has recently been demonstrated that is expressed in non-ciliated hematopoietic cells, and plays an important role in immune synapse assembly and intracellular membrane trafficking in T lymphocytes [9]. Additionally, our proteomic study has recently demonstrated that MIP-T3 can interact with different cytoskeletal proteins, chaperones, and signaling proteins, suggesting MIP-T3 may be an important protein involved in several cellular processes [10].

Cilia are highly conserved microtubule-based organelles that protrude from the surface of most eukaryotic cells. Cilia play diverse roles in cellular motility, sensory transduction and signaling processes critical in regulating vertebrate development and tissue homeostasis [11]. Defects in cilia structure or function lead to a wide range of human diseases and developmental disorders (ciliopathies) [12]. Accordingly, it can be considered that regulation of IFT protein stability is a key step in controlling the structure and function of cilia. Selective protein degradation is crucial for regulating a variety of basic cellular processes, including the control of cell signaling, regulation of cell cycle and division, modulation of the immune and inflammatory responses, and development and differentiation $[13,14]$. Rapid removal of cellular proteins is an important regulatory mechanism that can tightly control the expression and activity of a protein in eukaryotic cells. Thus, studying the degradation of IFT protein can provide valuable insights into understanding the regulation and cellular function of the protein. 
In this study, we investigated the stability of MIP-T3 protein in several human cell lines and found that MIP-T3 protein level is highly regulated; mainly mediated by the ubiquitin-proteasome system (UPS), and the C-terminal region is required for the UPS-mediated degradation of MIP-T3 protein.

\section{MATERIALS AND METHODS}

\subsection{Cell Cultures}

HEK293 and A549 cells were grown in DMEM (Invitrogen), HeLa cells were cultured in MEM (Invitrogen), and HSC-2 cells were maintained in MEM alpha (Invitrogen). All of the media were supplemented with 10\% fetal bovine serum and 1\% penicillin/streptomycin.

\subsection{Plasmid Constructs, Antibodies and Reagents}

Plasmids encoding human MIP-T3 gene and its deletion mutants, CL(aa211-625), $\mathrm{C}($ aa411-625) and NL(aa1-410), were generated by the polymerase chain reaction (PCR) using DNA polymerase KOD plus (TOYOBO), and then sub-cloned into pCAGGS or pEGFP-C1 vectors. Each plasmid DNA was purified by PureLink ${ }^{\text {TM }}$ HiPure Filter Plasmid Kit (Invitrogen) according to the manufacturer's instructions, and confirmed by restriction mapping and DNA sequencing. Guinea pig polyclonal antibody against MIP-T3 was generated as described previously [10]. Goat polyclonal anti-MIP-T3 antibody (Santa cruz), Rabbit monoclonal anti- $\beta$-actin antibody (Epitomics) and rabbit polyclonal anti-GFP antibody (MBL) were commercially purchased. MG132 was purchased from Calbiochem, and E64D, 3-Methyladenine (3-MA), and cycloheximide (CHX) were purchased from Sigma-Aldrich.

\subsection{RT-PCR}

For RT-PCR analysis, cells were dissolved by TRIzol LS reagent (Invitrogen), and total RNA was extracted. One microgram of total RNA was then reverse transcribed with Transcriptor Universal cDNA master (Roche) using random hexamer primers. MIP-T3 and GAPDH were respectively amplified by PCR using the following primers: forward, 5'-CCTGAAAGAACAAACGAGCTGCTCC-3'; and reverse, 5'-GTGAACTCTGGACTCTTCTTCTCGC-3', for MIP-T3 (expected length 
of product: 186bp); forward, 5'-AAGGTCGGAGTCAACGGATT-3'; and reverse, 5'-CTGGAAGATGGTGATGGGATT-3', for GAPDH (expected length of product: 222bp). The PCR was performed with the following conditions: 30 cycles of $95^{\circ} \mathrm{C}$ for $15 \mathrm{sec}$ and $65{ }^{\circ} \mathrm{C}$ for $1 \mathrm{~min}$. Five microliters of each RT-PCR product was electrophoresed on a $10 \%$ polyacrylamide gel followed by ethidium bromide staining.

\subsection{Western blotting and immunoprecipitation}

Cells were lysed and analyzed by Western blotting as described previously [10]. Beta-actin or alpha-tubulin was used as a loading control. Proteins were visualized by ECL detection reagent (GE Healthcare Biosciences). The protein level was determined by scanning of band intensity and analyzed using the IMAGEJ software (NIH).

For immunoprecipitation of ectopically expressed MIP-T3, a total of $5 \times 10^{6} \mathrm{HeLa}$ cells were transiently transfected with $10 \mu \mathrm{g}$ of pCAGGS-MIP-T3 plasmid using Lipofectamine 2000 (Invitrogen) for 24 hours, then treated with MG132 $(25 \mu \mathrm{M})$ or DMSO (vehicle control) for further 12 hours, subsequently performed immunoprecipitation as described previously [10]. Proteins were separated by $10 \%$ SDS-PAGE, blotted on PVDF membranes and analyzed by Western blotting. Ubiquitinated proteins were detected using mouse anti-mono- and poly-ubiquitinylated conjugate antibody (FK2H) (Enzo Life Sciences).

\subsection{Analysis of protein stability}

Cells were seeded in 24-well plate, transfected with $1 \mu \mathrm{g}$ of pCAGGS-MIP-T3 per well, and then incubated at $37^{\circ} \mathrm{C}$ for $24,48,72$ and 96 hours. Cells were harvested at the indicated time points, lysed and subjected to $10 \%$ SDS-PAGE, and analyzed by Western blotting using anti-MIP-T3 antibody and anti- $\beta$-actin antibody.

To analyze the stability of MIP-T3 protein and its truncation mutants, A549 cells were transiently transfected with MIP-T3 or its mutants for 18 hours, then further treated with $\mathrm{CHX}(100 \mu \mathrm{g} / \mathrm{ml})$ for 3, 6, 12, 18, 24 and 30 hours, respectively. Cells were lysed at the indicated time points and analyzed by Western blotting.

To examine the effect of MG132, E64D or 3-MA on degradation of MIP-T3 protein, MIP-T3-transfected A549 cells were treated with CHX $(100 \mu \mathrm{g} / \mathrm{ml})$ combined 
with a range of concentrations of MG132 $(5-50 \mu \mathrm{M})$, E64D $(25-200 \mu \mathrm{M})$ or 3-MA (5-25mM) for 18 hours, respectively, and then analyzed by Western blotting.

\section{RESULTS}

\subsection{Ectopically expressed MIP-T3 is rapidly degraded in human cells}

Since MIP-T3 mRNA has been demonstrated that is ubiquitously expressed in all of the tested tissues and cell lines in previous report [1], we first confirmed the mRNA and protein expression level of MIP-T3 in A549, HeLa and HSC-2 cells in this study. We found that the intrinsic MIP-T3 protein was hardly detected by Western blotting using specific anti-MIP-T3 antibody, although MIP-T3 mRNA is normally detectable (Fig. S1). These results suggest that steady-state level of MIP-T3 protein is kept extremely low or unstable in these human cell lines. Furthermore, we found that exogenously expressed MIP-T3 protein was rapidly decreased from 48 hours, and disappeared at 96 hours after transfection (Fig. 1A and B).

To confirm whether the degradation of MIP-T3 protein is due to the reduction of mRNA level, we investigated the MIP-T3 mRNA level using a semi-quantitative RT-PCR analysis. As shown in Fig. 1C and 1D, there was no significant time-dependent change in MIP-T3 mRNA expression in the transfected cells, suggesting the reduction of MIP-T3 protein was due to protein degradation rather than the change of mRNA level.

To further define the protein degradation is attributed to its protein nature, we compared the expression of GFP-fused MIP-T3 to GFP protein in human cells after transfection. As shown in Fig. 1E, GFP-fused MIP-T3 expressing cells were time-dependently reduced, but the GFP-expressing cells remain unchanged during 96 hours of transfecion. In addition, co-transfection of GFP and GFP-MIP-T3 also demonstrated that only GFP-MIP-T3 was specifically degraded in a time-dependent manner (Fig. 1F). These results strongly suggest that MIP-T3 is a relatively easily degradable protein in these cells. 


\subsection{Degradation of MIP-T3 is mediated by ubiquitin/proteasome pathway}

Generally, protein degradation in eukaryote cells is mediated by two major pathways, ubiquitin-proteasome pathway and autophagy-lysosome pathway [15]. To determine which proteolytic pathway is responsible for MIP-T3 degradation in human cell lines, we tested several protease inhibitors for their inhibitory effects on MIP-T3 protein degradation in A549 cells. As shown in Fig. 2A, treatment with the proteasome inhibitor MG132 restored the expressing level of MIP-T3 protein. In contrast, the cysteine protease inhibitor E64D, which inhibits calpain and lysosomal proteases, had no significant effect on MIP-T3 protein degradation. Interestingly, 3-Methyladenine (3-MA), an autophagy inhibitor, could partially rescue MIP-T3 protein degradation.

We further performed a CHX-chase assay by the addition of $\mathrm{CHX}$ combined with different concentrations of MG132, E64D and 3-MA to log-phase cultures followed by lysis at the indicated times. As shown in Fig. 2B and 2C, treatment with MG132, but not with E64D effectively inhibited MIP-T3 protein degradation in a dose-dependent manner. Whereas, 3-MA only partially inhibited the degradation treated at high concentration (Fig. 2D). These results suggest that MIP-T3 protein degradation is mainly mediated by proteasome degradation pathway.

Since polyubiquitination of protein is essential for proteasome-mediated proteolysis, we further confirmed that the effect of MG132 treatment on accumulation of polyubiquitinated cellular proteins. As shown in Fig. 2E, total polyubiquitinated proteins increased in the MG132-treated HeLa cells. Furthermore, immunoprecipitation experiment demonstrated that MIP-T3 protein level was increased by MG132 treatment (Fig. 2F), in which polyubiquitinated proteins were also accumulated (Fig. 2G).

\subsection{Endogenous MIP-T3 degradation is also mediated by ubiquitin/proteasome} pathway

To investigate whether the endogenous MIP-T3 is also easily degraded in human cells, we first examined the turnover of endogenous MIP-T3 by a CHX chase analysis 
combined with immunoprecipitation experiment. As showed in Fig 3A and 3B, after inhibition of protein synthesis by $\mathrm{CHX}$ treatment, the immunoprecipitated endogenous MIP-T3 protein level decreased in a time-dependent manner, suggesting that the endogenous MIP-T3 protein is rapidly degraded in these human cells.

We further confirmed the effects of MG132 on accumulation of endogenous MIP-T3 by a Western blotting/immunoprecipitation analysis. As shown in Fig. 3C, although the total polyubiquitinated proteins significantly accumulated in MG132-treated cells, but the endogenous MIP-T3 is still hardly detectable by Western blotting. Whereas, after enrichment by immunoprecipitation with anti-MIP-T3 antibody, the intrinsic MIP-T3 protein could be detected and was time-dependently increased following MG132 treatment, accompanying the increased levels of total polyubiquitinated protein in the cells (Fig. 3D). Taken together, these results demonstrated that the proteasome inhibition increased endogenous MIP-T3 protein level as well as elevated level of polyubiquitinated proteins, suggesting the intrinsic MIP-T3 protein degradation may be also mediated by ubiquitin/proteasome pathway.

\subsection{The C-terminus of MIP-T3 protein is crucial for its degradation}

To determine if there are conserved regions in MIP-T3 protein responsible for protein degradation and ubiquitination, we constructed several truncation mutants of MIP-T3 (Fig. 4A), and expressed them in HeLa cells (Fig. 4B). As shown in Fig. 4C, after CHX treatment, the expression levels of MIP-T3 and the mutants with the C-terminus (MIP-T3-CL and MIP-T3-C) significantly reduced in a time-dependent manner. In contrast, the mutant lacking C-terminal 215 amino acids (MIP-T3-NL) displayed resistance to degradation. The estimated half-life of exogenously expressed MIP-T3, MIP-T3-CL and MIP-T3-C protein were $21.7 \pm 2.7,14.8 \pm 1.1$ and $17.8 \pm 6.6$ hours, respectively, but that of MIP-T3-NL was 115 hours (Fig. 4D), indicating that the C-terminal region (residues 411 625) is crucial for mediating MIP-T3 protein degradation. 


\subsection{The C-terminal region of MIP-T3 is required for its ubiquitination}

To further confirm if the C-terminal region was required for ubiquitination of MIP-T3 protein, we expressed GFP-fused MIP-T3 (pEGFP-MIP-T3) and the GFP-fused truncation mutants in HeLa cells and then immunoprecipitated them using anti-GFP antibody. As shown in Fig 5A, GFP-fused MIP-T3 and its mutants were immunoprecipitated, respectively (upper panel). Wild-type MIP-T3, MIP-T3-CL and MIP-T3-C variants appeared to have significantly higher level of polyubiquitination than MIP-T3-NL (lower panel). Moreover, the relative ratios of bound polyubiquitinated proteins to GFP-MIP-T3 and its mutants were calculated respectively and summarized in Fig. 5B, deletion of C-terminal region of MIP-T3 (residues 411 and 625) abolished polyubiquitination of MIP-T3, suggesting the C-terminal is crucial for its polyubiqutination.

\section{DISCUSSION}

Recently, the ubiquitin-proteasome system (UPS) has been reported to be involved in the disassembly of cilia and flagella. During ciliary resorption, the ubiquitin conjugation system is present in cilia and adds an ubiquitin tag to axonemal turnover and breakdown products such as alpha-tubulin, which are transported by retrograde IFT complex to the cell body and can be reutilized in making new cilium [16]. However, whether the IFT proteins are also ubiquitinated during shortening process and the fates of these IFT proteins after ciliary resorption is still unclear. In mammalian cells, cilia and flagellar disassembly (also called resorption or shortening) occurs normally in synchrony with the cell cycle. Cilia must be removed before cell's entry into mitosis perhaps because the ciliary basal body must be freed from the cilium to become the centriole that helps form the mitotic spindle [17]. The present study for the first time showed that endogenously, or ectopically expressed MIP-T3 protein is indeed ubiquitinated and degraded via proteasome pathway in human cells, indicating the UPS may be involved in the regulation of IFT protein stability.

Notably, the C-terminal truncation inhibited the degradation and polyubiquitination of MIP-T3 protein, suggesting the C-terminus is required for its 
proteasomal degradation and polyubiquitination of MIP-T3. In most proteins, the preferred acceptor site for polyubiquitin chain addition is a lysine side chain. MIP-T3 sequence analysis confirmed that the C-terminus of MIP-T3 contains rich lysine residues, supporting the possibility that the major ubiquitin conjugation site is localized in the C-terminus of MIP-T3. Furthermore, the C-terminus also contains conserved coiled-coil domain, a protein-protein interacting interface [1], which is conserved in a number of IFT proteins (IFT81, IFT74/72, IFT57/55 and IFT20) [18]. Therefore, it is also possible the site is required for polyubiquitination mediated by E3 ubiquitin ligases, although the role of the conserved coiled-coil domain on regulation of IFT protein stability was not clarified in this study.

Moreover, multiple chaperone-assisted degradation pathways including CAP (chaperone-assisted proteasomal degradation), CASA (chaperone-assisted selective autophagy), and CMA (chaperone-mediated autophagy) have also been reported [19]. Within these pathways chaperones facilitate the sorting of non-native proteins to the proteasome and the lysosomal compartment and represent an essential aspect of cellular proteostasis. Since our previous study demonstrated MIP-T3 can interact with chaperones, including heat shock protein 90 (HSP90), heat shock $70 \mathrm{kDa}$ protein (HSP70), heat shock cognate $71 \mathrm{kDa}$ protein $(\mathrm{Hsc} 70)$, whether the interaction between MIP-T3 and these chaperones might contribute to its stability regulation via chaperone-assisted degradation needs further study. The present results show that 3-MA treatment had partial inhibitory effect on the MIP-T3 protein degradation, thus the CMA pathway may also partially involve in the degradation of exogenously expressed MIP-T3. However, more recently, 3-MA, the most commonly used pharmacologic inhibitor of autophagy, has been shown that has dual role in modulation of autophagy, there are marked increases of the autophagic markers in cells treated with 3-MA in full medium for a prolonged period of time (up to $9 \mathrm{~h}$ ), especially when used at high concentrations [20]. Therefore, the underlying mechanism of 3-MA treatment on MIP-T3 protein degradation needs further experiments to elucidate.

In conclusion, the present study revealed that MIP-T3 protein level in different 
human cell lines is regulated via the ubiquitin-proteasome degradation pathway. The C-terminal region of MIP-T3 is required for ubiquitination and the proteasome-dependent degradation of MIP-T3. This study provides the first evidence for regulation of IFT protein stability.

\section{ACKNOWLEDGEMENTS}

This work was partially supported by Grant-in-aid from the Tokyo Biochemical Research Foundation, a Grant-in-aid for Scientific Research from Japan Society for the Promotion of Science, and a Grant-in-Aid for Scientific Research (C) from the Ministry of Education, Culture, Sports, Science and Technology of Japan (No.22590274 to K.K.). 


\section{REFERENCE}

[1] Ling, L. and Goeddel, D.V. (2000). MIP-T3, a novel protein linking tumor necrosis factor receptor-associated factor 3 to the microtubule network. J Biol Chem 275, 23852-60.

[2] Niu, Y., Murata, T., Watanabe, K., Kawakami, K., Yoshimura, A., Inoue, J., Puri, R.K. and Kobayashi, N. (2003). MIP-T3 associates with IL-13Ralphal and suppresses STAT6 activation in response to IL-13 stimulation. FEBS Lett 550, 139-43.

[3] Morris, J.A., Kandpal, G., Ma, L. and Austin, C.P. (2003). DISC1 (Disrupted-In-Schizophrenia 1) is a centrosome-associated protein that interacts with MAP1A, MIPT3, ATF4/5 and NUDEL: regulation and loss of interaction with mutation. Hum Mol Genet 12, 1591-608.

[4] Inglis, P.N., Boroevich, K.A. and Leroux, M.R. (2006). Piecing together a ciliome. Trends Genet 22, 491-500.

[5] Kunitomo, H. and Iino, Y. (2008). Caenorhabditis elegans DYF-11, an orthologue of mammalian Traf3ip1/MIP-T3, is required for sensory cilia formation. Genes Cells 13, 13-25.

[6] Li, C. et al. (2008). An essential role for DYF-11/MIP-T3 in assembling functional intraflagellar transport complexes. PLoS Genet 4, e1000044.

[7] Omori, Y. et al. (2008). Elipsa is an early determinant of ciliogenesis that links the IFT particle to membrane-associated small GTPase Rab8. Nat Cell Biol 10, 437-44.

[8] Follit, J.A., Xu, F., Keady, B.T. and Pazour, G.J. (2009). Characterization of mouse IFT complex B. Cell Motil Cytoskeleton 66, 457-68.

[9] Finetti, F., Paccani, S.R., Riparbelli, M.G., Giacomello, E., Perinetti, G., Pazour, G.J., Rosenbaum, J.L. and Baldari, C.T. (2009). Intraflagellar transport is required for polarized recycling of the TCR/CD3 complex to the immune synapse. Nat Cell Biol 11, 1332-9.

[10] Guo, C.W. et al. (2010). Proteomic analysis reveals novel binding partners of MIP-T3 in human cells. Proteomics 10, 2337-47.

[11] Goetz, S.C. and Anderson, K.V. (2010). The primary cilium: a signalling centre during vertebrate development. Nat Rev Genet 11, 331-44.

[12] Fliegauf, M., Benzing, T. and Omran, H. (2007). When cilia go bad: cilia defects and ciliopathies. Nat Rev Mol Cell Biol 8, 880-93.

[13] Pedersen, L.B., Veland, I.R., Schroder, J.M. and Christensen, S.T. (2008). Assembly of primary cilia. Dev Dyn 237, 1993-2006.

[14] Ciechanover, A. (2005). Proteolysis: from the lysosome to ubiquitin and the proteasome. Nat Rev Mol Cell Biol 6, 79-87.

[15] Kraft, C., Peter, M. and Hofmann, K. (2010). Selective autophagy: ubiquitin-mediated recognition and beyond. Nat Cell Biol 12, 836-41.

[16] Huang, K., Diener, D.R. and Rosenbaum, J.L. (2009). The ubiquitin conjugation system is involved in the disassembly of cilia and flagella. J Cell Biol 186, 601-13.

[17] Quarmby, L.M. and Parker, J.D. (2005). Cilia and the cell cycle? J Cell Biol 169, 707-10.

[18] Cole, D.G. (2003). The intraflagellar transport machinery of Chlamydomonas reinhardtii. Traffic 4, 435-42.

[19] Kettern, N., Dreiseidler, M., Tawo, R. and Hohfeld, J. (2010). Chaperone-assisted degradation: multiple paths to destruction. Biol Chem 391, 481-9.

[20] Wu, Y.T. et al. (2010). Dual role of 3-methyladenine in modulation of autophagy via different temporal patterns of inhibition on class I and III phosphoinositide 3-kinase. J Biol Chem 285, 10850-61. 


\section{Figure legends}

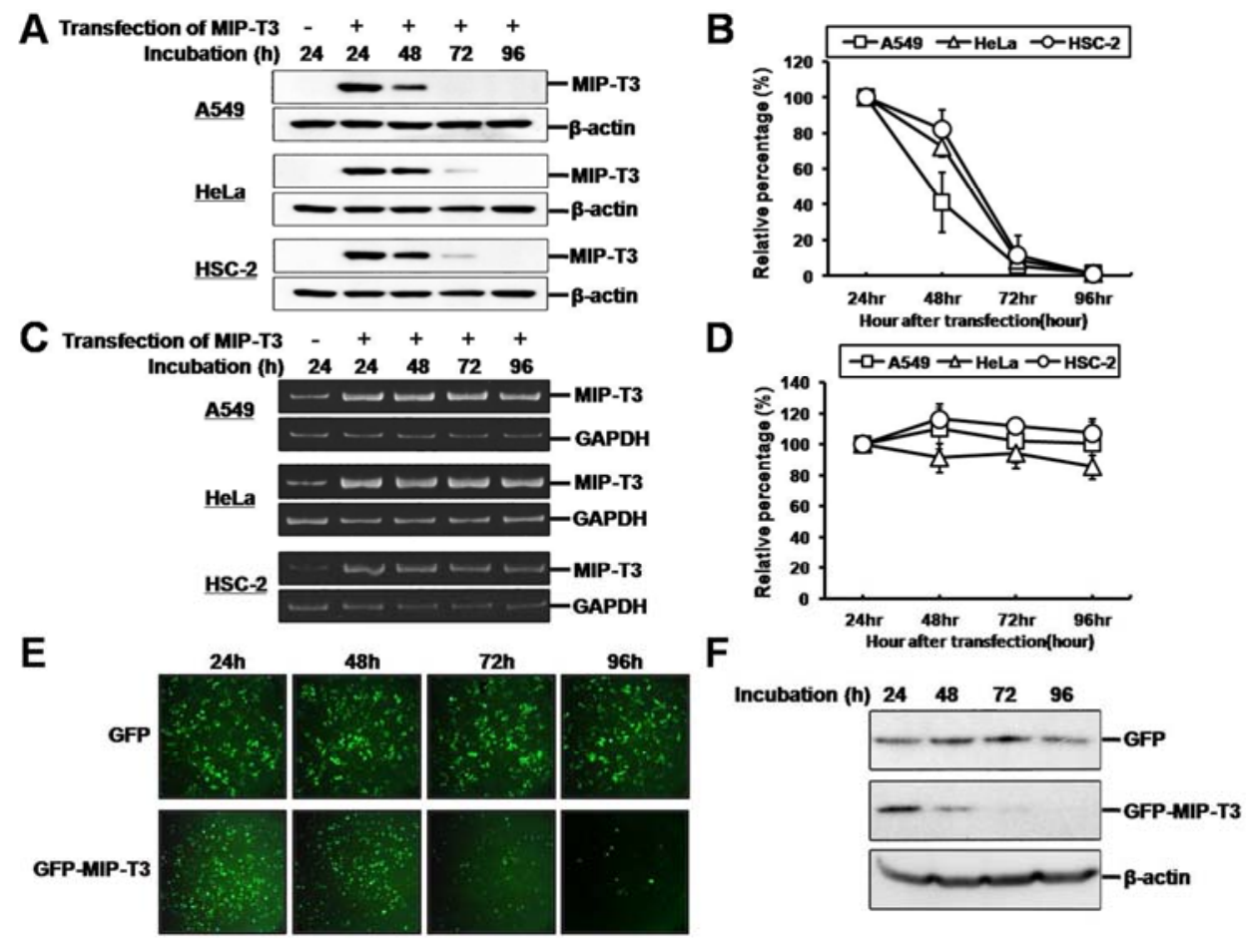

Fig. 1 Time-dependent degradation of MIP-T3 protein in different human cells

(A) Western blot analysis for expressions of MIP-T3 and $\beta$-actin in A549, HeLa and HSC-2 cells, which was transiently transfected with pCAGGS-MIP-T3 for 24, 48, 72 and 96 hours, respectively. (B) Relative percentage of MIP-T3 level was time-dependently decreased during 96 hours of transfection. (C) Total cellular RNA was extracted from transfected cells, and the mRNA levels of MIP-T3 and GAPDH were respectively analyzed by RT-PCR. (D) No significant time-dependent changes in the MIP-T3 mRNA level was observed from 24 to 96 hours after transfection (E) Fluorescence microscopic observations of GFP and GFP-fused MIP-T3 expressed in A549 cells at the indicated time point after transfection. (F) Western blot analysis for GFP, GFP-fused MIP-T3, and $\beta$-actin in A549 cells at the indicated time point after co-transfection. 


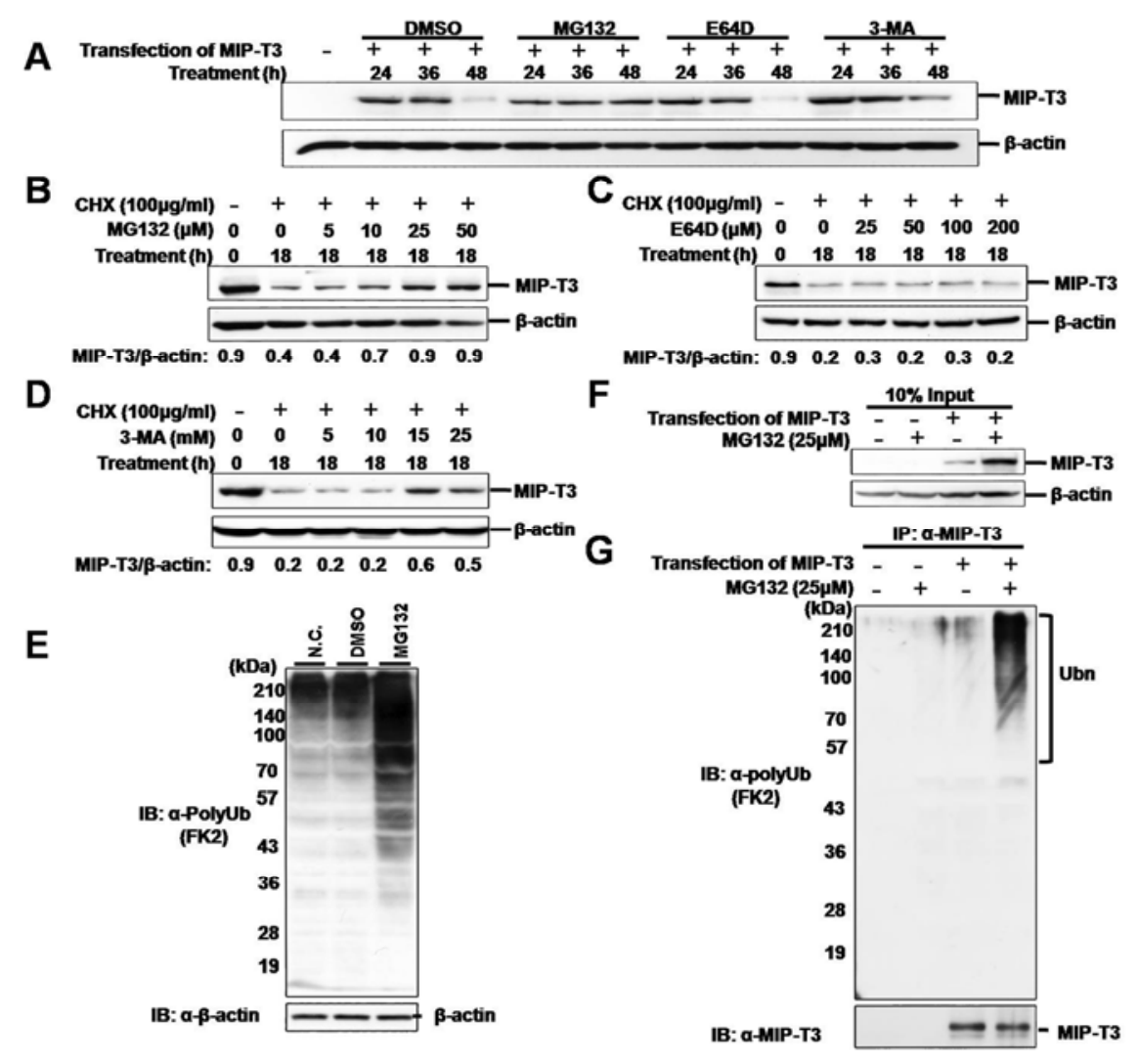

Fig. 2 MIP-T3 protein degradation was mediated by ubiquitin-proteasome pathway

(A) Effects of various inhibitors treatment on MIP-T3 protein degradation. A549 cells were transiently transfected with pCAGGS-MIP-T3 and treated with various protease inhibitors, MG132 $(25 \mu \mathrm{M}), \mathrm{E} 64 \mathrm{D}(25 \mu \mathrm{M})$ and 3-MA $(10 \mathrm{mM})$ for the indicated period. DMSO was used as a

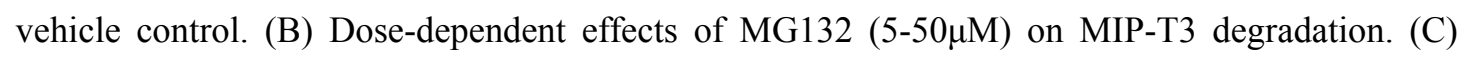

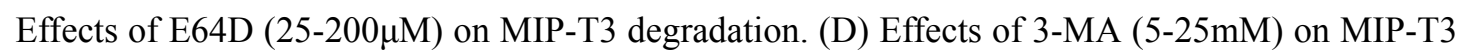
degradation. (E) Total cellular polyubiquitinated proteins are accumulated following 12 hours of treatment with MG132 $(25 \mu \mathrm{M})$ and DMSO $(0.1 \% \mathrm{v} / \mathrm{v}$, vehicle control). (F and G) MIP-T3 protein level increased in MG132-treated cells. Immunoprecipitation/Western blot analyses showed that MIP-T3 protein was polyubiquitinated in MG132-treated cells. The asterisk indicates IgG heavy chain, and the bracket indicates polyubiquitinated proteins. 
A

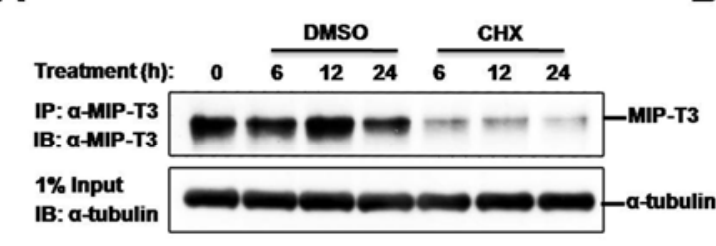

C

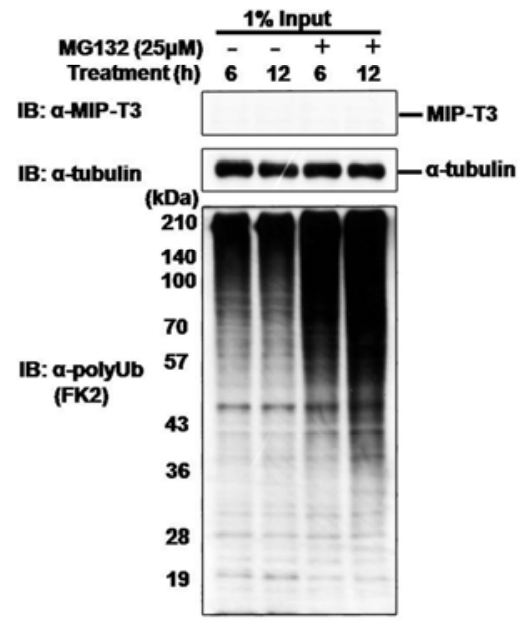

B

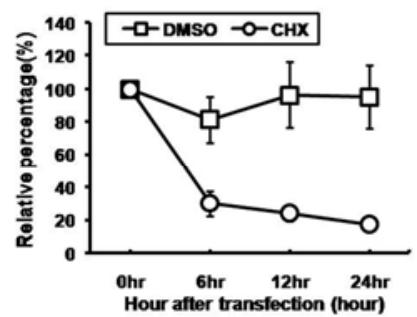

D

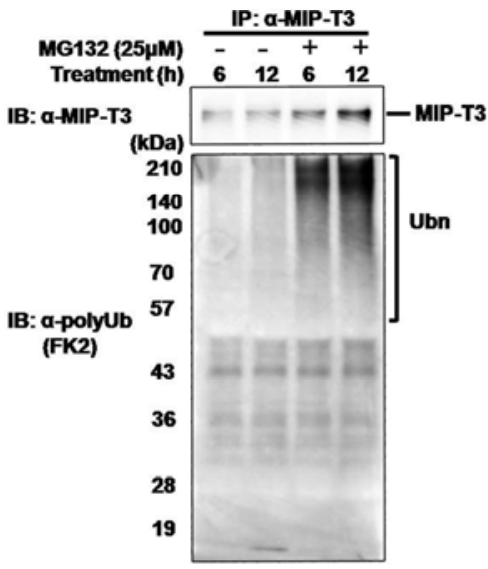

Fig. 3 Intrinsic MIP-T3 degradation was mediated by ubiquitin-proteasome pathway

(A) CHX-chase analysis of the intrinsic MIP-T3 turnover. Intrinsic MIP-T3 protein in DMSO or CHX treated HeLa cells were immunoprecipitated at the indicated time point and subject to Western blot analysis using anti-MIP-T3 antibody. Alpha-tubulin in each cell lysate was also detected as the loading control. (B) Relative percentage of the amount of intrinsic MIP-T3 protein normalized by $\alpha$-tubulin protein level at the indicated time point relative to that at $0 \mathrm{hr}$. Error bars indicate mean values \pm SD from three independent experiments. (C) Accumulation of the

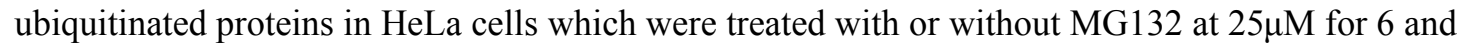
$12 \mathrm{hr}$. Total cell lysates were prepared, and $1 \%$ of input was analyzed by Western Blotting. (D) The remaining lysates were analyzed by immunoprecipitation/Western blotting. MG132 treatment time-dependently increased the accumulation of endogenous MIP-T3 proteins as well as the elevated level of polyubiquitinated proteins. Ubn indicates polyubiquitinated proteins. 
A

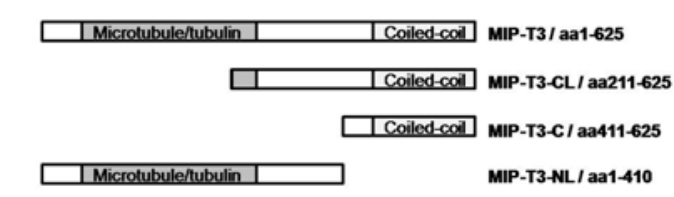

C

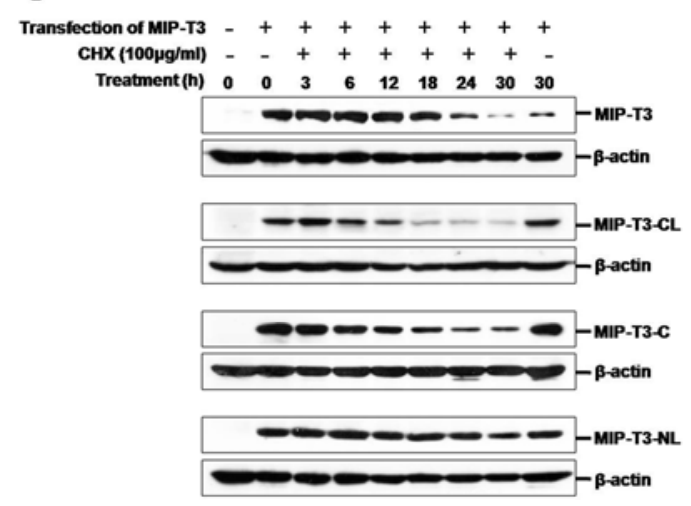

B

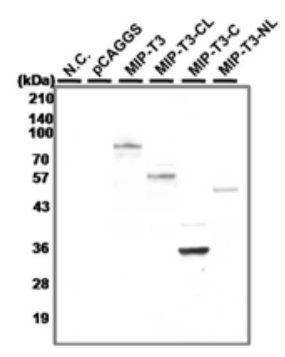

D

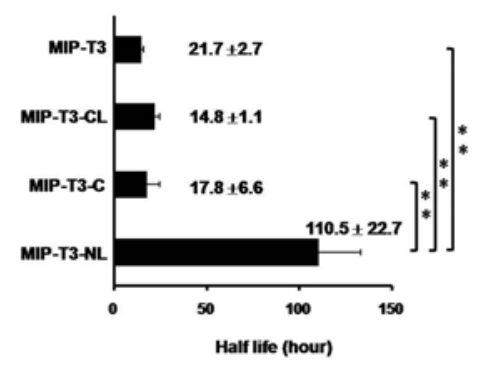

Fig. 4 C-terminal region was crucial for degradation of MIP-T3 protein in cells

(A) The schematic representation of MIP-T3 and its truncation mutants. (B) Western blot analysis of expressions of MIP-T3 and its mutants in A549 cells at 24 hours post-transfection. (C) Expressions of MIP-T3 and its mutants in A549 cells treated with CHX $(100 \mu \mathrm{g} / \mathrm{mL})$ for the indicated period after 18 hours of transfection. (D) The estimated half-life of each protein was showed in lower panel. Data were presented as the means $\pm \mathrm{SD}$ of three independent experiments. The asterisks indicate significant differences in protein expressing levels between the MIP-T3 NL and the others, $* \mathrm{P}<0.05, * * \mathrm{P}<0.01$. 
A

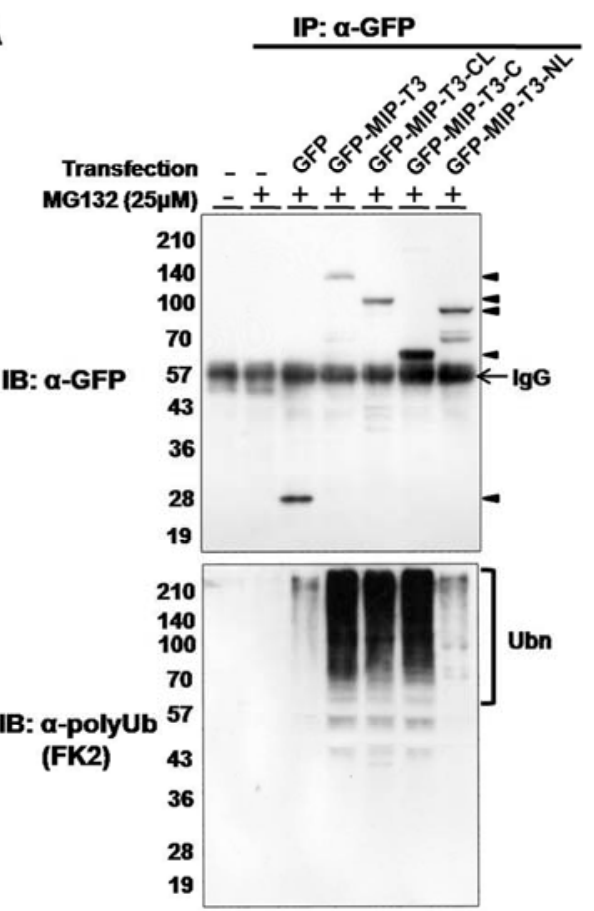

B

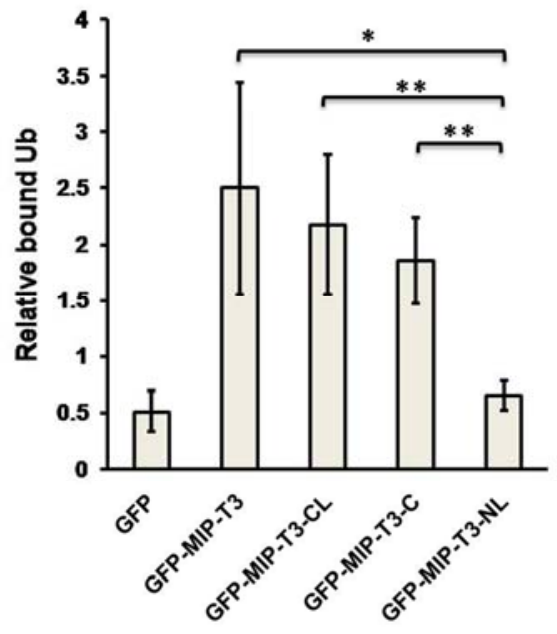

Fig. 5 C-terminal region of MIP-T3 was required for ubiquitination.

(A) HeLa cells were transfected with pEGFP, GFP-MIP-T3 and its GFP-tagged truncation mutants for 24 hours, respectively, and treated with MG132 for a further 12 hours, and then immunoprecipitated with polyclonal anti-GFP antibody. The immunoprecipitates were analyzed by Western blotting using anti-GFP (upper panel) and anti-ubiquitin (lower panel) antibodies. Ubn indicates polyubiquitinated proteins. The closed arrowheads indicate the position of each protein. (B) The relative ratio of bound multi-ubiquitinated proteins to GFP-MIP-T3 and its mutants. The asterisks indicate significant differences in relative protein levels between the MIP-T3 NL and the others, $* \mathrm{P}<0.05, * * \mathrm{P}<0.01$. 
Fig. S1 MIP-T3 mRNA is ubiquitously expressed but its steady-state protein level is hardly detectable by Western blotting.

(A) Total RNA was extracted from A549, HeLa and HSC-2 cells, and the mRNA levels of MIP-T3 and GAPDH were analyzed by RT-PCR, respectively. (B) Western blotting was performed using anti-MIP-T3 antibody, demonstrating that an $83 \mathrm{kDa}$ band in the positive control HeLa cells, which were transiently transfected with pCAGGS-MIP-T3, was not detected in all the examined cell lines without exogenously transfection of MIP-T3.

A

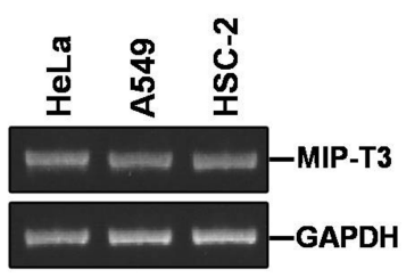

B

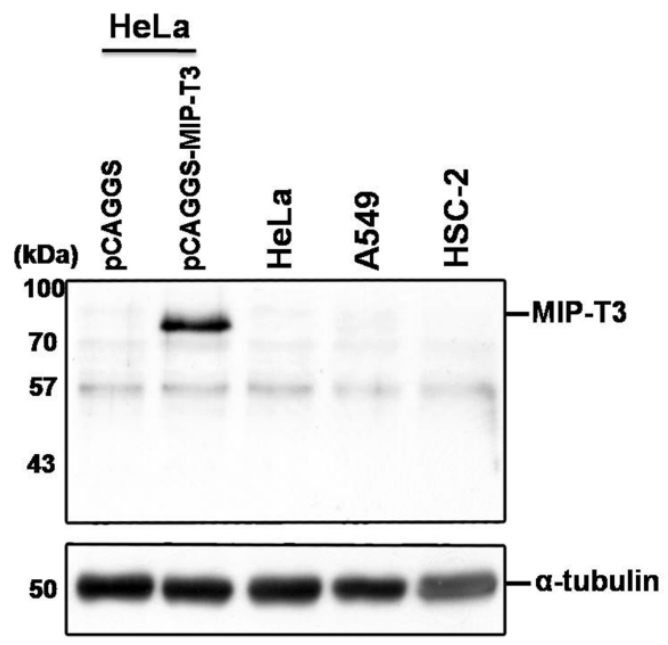




\section{Highlights}

$>$ In this study we examine a novel IFT protein MIP-T3's protein stability in human cells. $>$ We found that endogenously, or ectopically expressed MIP-T3 protein is unstable. >The protein degradation is mediated by the ubiquitin-proteasome system. > The C-terminal is required for its ubiquitination and proteasome-mediated protein degradation. > We provide the first evidence for regulation of IFT protein stability. 